\title{
ADER schemes and high order coupling on networks of hyperbolic conservation laws
}

\author{
Raul Borsche ${ }^{\mathrm{a}, *}$, Jochen Kall ${ }^{\mathrm{a}}$ \\ ${ }^{a}$ Erwin Schrödinger Straße, TU Kaiserslautern, Building 48, 67663 Kaiserslautern, \\ Germany
}

\begin{abstract}
In this article we present a method to extend high order finite volume schemes to networks of hyperbolic conservation laws with algebraic coupling conditions. This method is based on an ADER approach in time to solve the generalized Riemann problem at the junction. Additionally to the high order accuracy, this approach maintains an exact conservation of quantities if stated by the coupling conditions. Several numerical examples confirm the benefits of a high order coupling procedure for high order accuracy and stable shock capturing.
\end{abstract}

Keywords: ADER, Network, hyperbolic conservation law, WENO, generalized Riemann problem, Coupling

\section{Introduction}

Networks of hyperbolic conservation laws occur in many applications such as the human circulatory system $[1,2,3,4]$, gas pipelines $[5,6,7]$, water $[8,9,10,11]$ and road networks $[12,13]$. For all these applications accurate and stable numerical methods are needed.

In the past decades high order accurate numerical methods for hyperbolic conservation laws have been developed, such as WENO- [14, 15, 16] or ADER-schemes $[17,18,19,20]$. These methods have proven their efficiency in many challenging applications $[21,22]$.

\footnotetext{
*Corresponding author

Email addresses: borsche@mathematik.uni-kl.de (Raul Borsche), kall@mathematik.uni-kl.de (Jochen Kall)
} 
For networks of hyperbolic conservation laws the flow across the edges can be dealt with by any appropriate numerical method for standard conservation laws [18]. Special attention has to be given to the coupling conditions. The direct solving of the coupling conditions only provides first order information, which can either be used directly by applying a Godunov scheme [6, 23] or to fill corresponding ghost cells $[9,24]$ at the boundary. A second order approach is studied in [25].

In this article we present an approach to incorporate the coupling conditions numerically up to an arbitrary order of accuracy. This includes the computation of the flux across the outer boundary as well as the reconstruction of ghost cell values for numerical methods of higher order. Therefore we apply an ADER approach in time to the algebraic coupling conditions. This can be used to solve the generalized Riemann problems at the junctions providing time dependent data at the junction. These can be reformulated as spatial data by the inverse Cauchy-Kowalewski procedure to fill the ghost cells for the numerical method along the edges.

This paper is organized as follows. First, the first order Godunov solver at the junction is recalled. Second, the generalized Riemann Problem at the junction is discussed. These two ingedients can be used for high order spatial reconstruction at the nodes, which leads to a high order numerical method for the complete network. For this approach we prove that the quantities conserved by the algebraic coupling conditions are also conserved by the numerical method. Further we show that for a simple 1 to 1 coupling the presented method coincides with a classical ADER scheme on a single continuous line. In the numerical examples we study the order accuracy for test cases with smooth data and show the need of a high order coupling procedure. Finally we investigate the stability in case of shock waves and the applicability for large networks of conservation laws.

\section{High order coupling procedure}

\subsection{Notations}

A network $\mathcal{N}=(\mathcal{E}, \mathcal{V})$ consists of a set of edges $\mathcal{E}$ and a set of connecting vertices $\mathcal{V}$

$$
\mathcal{E}=\left\{E_{1}, \ldots, E_{\tilde{n}}\right\}, \quad \mathcal{V}=\left\{V_{1}, \ldots, V_{\tilde{m}}\right\}
$$


On each edge $E_{i}, i=1, \ldots, \tilde{n}$, we consider the quantities $u^{i}(x, t) \in \mathbb{R}^{d_{i}}$, which are governed by a hyperbolic conservation law

$$
\partial_{t} u^{i}+\partial_{x} f^{i}\left(u^{i}\right)=0
$$

with the flux function $f^{i}: \mathbb{R}^{d_{i}} \rightarrow \mathbb{R}^{d_{i}}$, the time $t \in \mathbb{R}^{+}$and location $x \in\left[0, L_{i}\right]$. At every vertex $v_{j}$, the functions $u^{i}$ are coupled via $c^{j}$ algebraic coupling conditions given by $\Phi^{j}: \bigotimes_{i=1}^{n} \mathbb{R}^{d_{i}} \rightarrow \mathbb{R}^{c^{j}}$ for $n$ connected edges. In order to ease the notation in the following we consider only a single junction without index and assume that all $n=\tilde{n}$ connected edges are oriented outwards. Thus the coupling point in each edge is located at $x=0$ allowing us to drop the spatial variable in the context of the coupling conditions

$$
\Phi\left(u^{1}(t), \ldots, u^{n}(t)\right)=0, \quad u^{i}(t)=u^{i}(0, t) .
$$

Following the results of $[26,9]$, the number of coupling conditions $c$ has to coincide with the number of characteristics running out of the vertex. In order to maintain a fixed number of coupling conditions over time, we require for each edge $i$ the eigenvalues $\lambda_{j}^{i}, j=1, \ldots, d_{i}$, of the Jacobian $\nabla F^{i}$ to be bounded away from zero by some constant $\tilde{\epsilon}>0$

$$
\lambda_{1}^{i} \leq \ldots \leq \lambda_{d_{i}}^{i}, \quad\left|\lambda_{j}^{i}\right|>\tilde{\epsilon} \quad \forall j=1, \ldots, d_{i} .
$$

Finally the following condition guarantees that to each outgoing characteristic exactly one value can be assigned and thus the well-posedness of the coupling conditions is given by

$$
\operatorname{det}\left(D_{u^{1}} \Phi\left(u_{g}^{1}, \ldots, u_{g}^{n}\right) R^{1}|\ldots| D_{u^{n}} \Phi\left(u_{g}^{1}, \ldots, u_{g}^{n}\right) R^{n}\right) \neq 0,
$$

where $R^{i}=\left[r_{d_{i}-c_{i}+1}^{i}|\ldots| r_{d_{i}}^{i}\right]$ is the collection of all eigenvectors associated with positive eigenvalues of $\nabla f^{i} . c_{i}$ is the number of positive eigenvalues in the edge $i$ and $\sum_{i=1}^{n} c_{i}=c$ holds.

\subsubsection{Examples}

Throughout this paper we will consider the isentropic Euler equations as example

$$
\begin{aligned}
\partial_{t} \rho+\partial_{x} q & =0 \\
\partial_{t} q+\partial_{x}\left(\frac{q^{2}}{\rho}+p(\rho)\right) & =0
\end{aligned}
$$


with the density $\rho$, density flux $q$ and a pressure law $p: \mathbb{R}^{+} \rightarrow \mathbb{R}^{+}$. Commonly used coupling conditions in this context for subsonic flow, $\frac{q}{\rho}<\sqrt{\partial_{\rho} p(\rho)}$, are the following two variants.

Definition 1. Pressure coupling [6, 24]:

$$
\begin{gathered}
\sum_{i=1}^{n} q_{i}=0 \\
p_{1}\left(\rho_{1}\right)-p_{i}\left(\rho_{i}\right)=0 \quad 2 \leq i \leq n .
\end{gathered}
$$

In case of an identical pressure law in all connected edges, the last $n-1$ equations reduce to $\rho_{1}-\rho_{i}=0 \quad 2 \leq i \leq n$.

Definition 2. Impluse-flux coupling [27, 28]:

$$
\begin{gathered}
\sum_{i=1}^{n} q_{i}=0 \\
\frac{q_{1}^{2}}{\rho_{1}}+p_{1}\left(\rho_{1}\right)-\frac{q_{i}^{2}}{\rho_{i}}-p_{i}\left(\rho_{i}\right)=0 \quad 2 \leq i \leq n .
\end{gathered}
$$

In both cases the first coupling condition ensures the conservation of mass across the junction. In case of a 2 -junction, i.e. $n=2$, these conditions ensure the continuity of the states across the junction, i.e. $u_{1}(0, t)=u_{2}(0, t)$.

\subsection{The classical Riemann problem at the junction}

The Riemann problem at a junction is defined by (1) and (2) with given constant initial data on the edges

$$
u^{i}(x, 0)=u_{r}^{i} \quad i=1, \ldots, n .
$$

In case of a 2-junction and for suitable coupling conditions, e.g. (6) or (7), a classical Riemann problem is obtained. A detailed analysis of the Riemann problem at a junction of $p$-systems can be found in [29]. In the following we consider this approach in the context of general systems of hyperbolic conservation laws. For the solution at the junction the Godunov states of the Riemann problems at the interfaces play a key role. All subsonic Godunov 


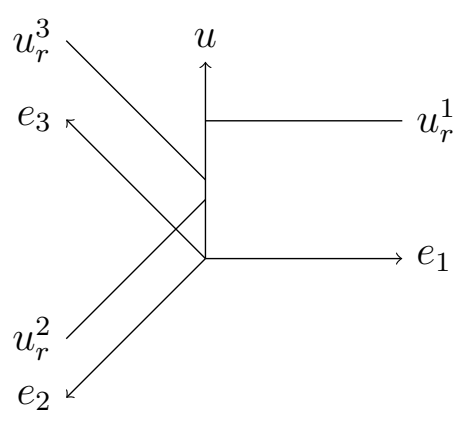

(a) Classical Riemann problem at the junction

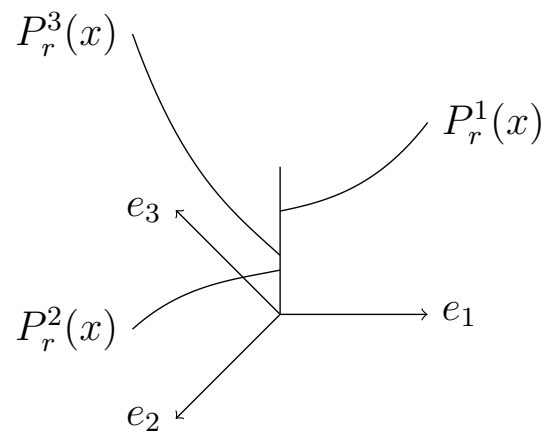

(b) Generalized Riemann problem at the junction

Figure 1: Riemann problems at a 3-way junction.

states, which can be connected by waves going into the edge, lie on the concatenation of the Lax-curves corresponding to the positive eigenvalues,

$$
L_{g}^{i}\left(\xi_{1}^{i}, \ldots, \xi_{c_{i}}^{i}, u_{r}^{i}\right)=L_{d_{i}-c_{i}+1}\left(\xi_{1}^{i}, \cdot\right) \circ \ldots \circ L_{d_{i}}^{i}\left(\xi_{c_{i}}^{i}, u_{r}^{i}\right)
$$

where $c_{i}=\#\left\{\lambda_{j}^{i} \mid \lambda_{j}^{i}>0\right\}$. Here $\circ$ denotes the concatenation in the last variable, i.e for two functions $g$ and $h$

$$
g\left(\xi_{1}, \ldots, \xi_{l}, \cdot\right) \circ h\left(\xi_{l+1}, \ldots, \xi_{m}, x\right)=g\left(\xi_{1}, \ldots, \xi_{l}, h\left(\xi_{l+1}, \ldots, \xi_{m}, x\right)\right)
$$

The Lax-curves describe all reachable subsonic states, thus we can use the coupling conditions to determine the unique states at the junction, i.e. we solve the following system of nonlinear equations

$$
\Phi\left(L_{g}^{1}\left(\xi^{1}, u_{r}^{1}\right), L_{g}^{2}\left(\xi^{2}, u_{r}^{2}\right), \ldots, L_{g}^{n}\left(\xi^{n}, u_{r}^{n}\right)\right)=0
$$

for the unknowns $\xi^{i}=\left(\xi_{1}^{i}, \ldots, \xi_{c_{i}}^{i}\right)$. The local solvability of this system is assured by condition (4). Once the parameters $\xi^{i}$ are known, the Godunov states at the interfaces can be determined by evaluating the concatenated Lax-curves

$$
u_{g}^{i}=L_{g}^{i}\left(\xi^{i}, u_{r}^{i}\right) .
$$

This construction guarantees that the states at the junction are reached only by waves traveling into the edges and no information is lost due to waves exiting the domain. 


\subsection{The generalized Riemann problem at the junction}

Analogously to the classical generalized Riemann problem, we formulate the generalized Riemann problem at the junction. Consider the equations (1) and (2) equipped with polynomial initial data on each edge

$$
u^{i}(x, 0)=\sum_{k=0}^{k_{\max }} \frac{\partial_{x}^{k} u_{r}^{i}}{k !} x^{k} \quad i=1, \ldots, n,
$$

for a fixed $k_{\max } \in \mathbb{N}$. Again, this reverts to the classical generalized Riemann problem for $n=2$ and a suitable choice of coupling conditions. In order to solve the generalized Riemann problem at the junction, we use an ADER approach [18]. Therefore we split the problem into several subproblems, one for each degree of the polynomials chosen as initial data. For the terms of order zero we solve a classical Riemann problem at the junction with the initial data

$$
u^{i}(x, 0)=\partial_{x}^{0} u_{r}^{i}
$$

The corresponding Godunov states $u_{g}^{i}$ are computed following the construction of section 2.2. For the higher order terms we obtain the governing equations by differentiating (1) w.r.t. $t$ repeatedly

$$
\partial_{t}\left(\partial_{t}^{k} u^{i}\right)+\nabla f^{i}\left(u_{g}^{i}\right) \partial_{x}\left(\partial_{t}^{k} u^{i}\right)+\text { 'sources' }=0 \quad k=1, \ldots, k_{\max } .
$$

The term 'sources' contains all contributions of derivatives with degree less than $k$. These can be dropped, since only the first instant interaction is relevant for the solution of a Riemann problem [18]. The governing equations for the temporal derivatives are linear and therefore the corresponding Lax curves are linear as well. The concatenated Lax curves have the short form

$$
L_{g}^{i}\left(\xi^{i}, u_{r}\right)=u_{r}+R^{i} \xi^{i}, \quad \quad R^{i}=\left[r_{d_{i}-c_{i}+1}^{i}|\ldots| r_{d_{i}}^{i}\right],
$$

where $r_{j}^{i}$ denotes the eigenvector corresponding to the $j$-th eigenvalue $\lambda_{j}^{i}$ of $\nabla f^{i}\left(u_{g}^{i}\right)$. Note that (3) still holds as the Jacobian is the same as in the Riemann problem of order zero.

As the states in the junction may vary in time, they have to fulfill the coupling conditions at all times. Therefore all temporal derivatives of the coupling conditions at $t=0$ have to vanish. The first order derivative of $\Phi$ 
reads

$$
\begin{aligned}
\Phi\left(u^{1}, \ldots, u^{n}\right) & =0 \\
\Rightarrow \partial_{t} \Phi\left(u^{1}, \ldots, u^{n}\right) & =0 \\
\Rightarrow \sum_{l=1}^{n} D_{u^{l}} \Phi\left(u^{1}, \ldots, u^{n}\right) \partial_{t} u^{l} & =0
\end{aligned}
$$

where all quantities are evaluated at $t=0$ and $x=0$. Here and in the following we assume $\Phi$ to be sufficiently differentiable, otherwise we consider the usage of high order schemes not appropriate. For the derivatives of orders $k \geq 2$ additional terms arise, but it is easy to see that these only depend on derivatives of lower order,

$$
D \Phi\left(u_{g}^{1}, \ldots, u_{g}^{n}\right) \partial_{t}^{k} u+\Psi_{k}\left(u_{g}, \partial_{t} u_{g}, \ldots, \partial_{t}^{k-1} u_{g}\right)=0 .
$$

Note that these lower order terms $\Psi_{k}$ can not be dropped as in (9), but all the information needed is available from previous steps. Therefore the resulting linear systems have to be solved successively in increasing order of the derivatives. As in the zeroth order case, we now insert the concatenated linear Lax-curves (10)

$$
\begin{array}{r}
\sum_{i=1}^{n} D_{u^{i}} \Phi\left(u_{g}^{1}, \ldots, u_{g}^{n}\right)\left(R^{i} \xi_{k}^{i}+\partial_{t}^{k} u_{r}^{i}\right)+\Psi_{k}=0 \\
\Rightarrow \sum_{i=1}^{n} D_{u^{i}} \Phi\left(u_{g}^{1}, \ldots, u_{g}^{n}\right) R^{i} \xi_{k}^{i}+\sum_{i=1}^{n} D_{u^{i}} \Phi\left(u_{g}^{1}, \ldots, u_{g}^{n}\right) \partial_{t}^{k} u_{r}^{i}+\Psi_{k}=0 .
\end{array}
$$

By introducing the notations

$$
\begin{aligned}
a_{i} & =D_{u^{i}} \Phi\left(u_{g}^{1}, \ldots, u_{g}^{n}\right) R^{i}, & A & =\left(a_{1}\left|a_{2}\right| \ldots \mid a_{n}\right), \\
\xi_{k} & =\left(\begin{array}{lllllll}
\xi_{k}^{1} & \xi_{k}^{2} & \ldots & \xi_{k}^{n}
\end{array}\right)^{T}, & \partial_{t}^{k} u_{r} & =\left(\begin{array}{lllll}
\partial_{t}^{k} u_{r}^{1} & \partial_{t}^{k} u_{r}^{2} & \ldots & \partial_{t}^{k} u_{r}^{n}
\end{array}\right)^{T}
\end{aligned}
$$

this linear system can be written in the form

$$
A \xi+D \Phi\left(u_{g}^{1}, \ldots, u_{g}^{n}\right) \partial_{t}^{k} u_{r}+\Psi_{k}=0 .
$$

The matrix $A$ is exactly the one in (4) considered for the well-posedness of the coupling conditions. Since we have $\operatorname{det} A \neq 0$, we can solve for the unknowns $\xi_{k}$

$$
\xi_{k}=A^{-1}\left(-D \Phi\left(u_{g}^{1}, \ldots, u_{g}^{n}\right) \partial_{t}^{k} u_{r}-\Psi_{k}\right)
$$


In order to evaluate this expression, the temporal derivatives of the states within the edges $\partial_{t}^{k} u_{r}$ are needed. These can be obtained from the given spatial derivatives $\partial_{x}^{k} u_{r}$ using the Cauchy-Kowalewski or Lax-Wendroff procedure $[18,30]$. Note that it is important that the Cauchy-Kowalewski procedure is carried out on the basis of the zeroth order Godunov state, which can be obtained from the classical Riemann Problem at the junction. Here we use the following modified Cauchy Kowalewski procedure

$$
\partial_{t}^{k} u_{r}^{i}=\mathrm{CK}\left(\mathrm{CK}^{-1}\left(u_{g}^{i}, \partial_{t} u_{g}^{i}, \ldots, \partial_{t}^{k-1} u_{g}^{i}\right), \partial_{x}^{k} u_{r}^{i}\right),
$$

where CK denotes the classical Cauchy Kowalewski procedure translating spatial derivatives into temporal ones and $\mathrm{CK}^{-1}$ its inverse performing the backward transformation. This modified procedure requires a successive solving of the coupling conditions increasing in the order of the derivatives, i.e. for the order $k$ all the temporal derivatives of the Godunov states $u_{g}^{i}$ of order less than $k$ have to be known. Alternatively the approach in [31] seems to be suitable as well.

\subsection{Spatial reconstruction at the junction}

In this section we address the spatial reconstruction at the node. In fact we have to consider two separate problems, the reconstruction for the

numerical scheme within the edges and the reconstruction for the generalized Riemann problem at the junction.

\subsubsection{Reconstruction for the numerical scheme}

Assume we want to update the solutions within the edges from time step $t_{l}$ to $t_{l+1}$. Further we assume that we have sufficient initial data given for the generalized Riemann problem at the junction. Then we can compute the temporal evolution of the states at the junction according to section 2.3, i.e. we obtain for each connected edge $i=1, \ldots, n$ a polynomial in $\tau$

$$
u_{g}^{i, l}\left(t_{l}+\tau\right)=\sum_{j=0}^{k_{\max }} \frac{\partial_{t}^{j} u_{g}\left(t_{l}\right)}{j !} \tau^{j} .
$$

Each of these polynomials provide sufficient information for a numerical scheme along the corresponding edge. Firstly it can be used to evaluate the flux across the interface at the junction. Therefore we directly compute 
the flux across the interface with the formula of the ADER approach [18]

$$
F_{1 / 2}=\left.\frac{1}{\Delta t} \int_{0}^{\Delta t} \sum_{k=0}^{k_{\max }} \frac{\tau^{k}}{k !}\left[\partial_{t}^{k} f^{i}\left(u_{g}^{i}(\tau)\right)\right]\right|_{\tau=0} d \tau
$$

For this expression Taylor approximation of $f$ should be used. An approximation via a quadrature formula can violate the exact conservation property at the junction, see section 2.6.

Secondly the polynomial (13) can be used to fill the ghost cells at the junction, as the cell interfaces in the wider neighborhood of the junction still require spatial information. This can be achieved via the inverse CauchyKowalewski procedure, which converts the temporal polynomial into a spatial one. From this spatial polynomial the data for the ghost cells is simply obtained by integration.

\subsubsection{Reconstruction for the generalized Riemann problem at the junction}

For the generalized Riemann problem at the junction we need spatial polynomials at the first interface of each edge as input data. These polynomials are obtained via classical WENO reconstruction [14], which is allowed to access values in the ghost cells at the junction. In the following we describe how to fill these ghost cells with appropriate data.

We assume that the numerical scheme already has performed the update from $t_{l}$ to $t_{l+1}$. If there have been no computations before we fill the ghost cells with data obtained by a one sided reconstruction presented in [30]. If the computations from time step $t_{l}$ are available, we can simply use the polynomial (13). In the previous update it was used to describe the Godunov state at the junction during the period $\left[t_{l}, t_{l+1}\right]$. Now we use it to fill the ghost cells at the time level $t_{l+1}$. For that purpose, we transform the polynomial

$$
u_{g}^{i, l}\left(t_{l}+\Delta t+\tau\right)=\sum_{j=0}^{k_{\max }} \frac{\partial_{t}^{j} u_{g}\left(t_{l}\right)}{j !}(\Delta t+\tau)^{j}
$$

via inverse Cauchy Kowalewski procedure into a spatial polynomial and compute the cell averages. A schematic illustration is given in figure 2. In formula (15) it seems that $u_{g}^{i, l}$ is used over its livetime $t_{l+1}$ given by the CFL condition, but it is only used to construct spatial data at $t_{l+1}$. 


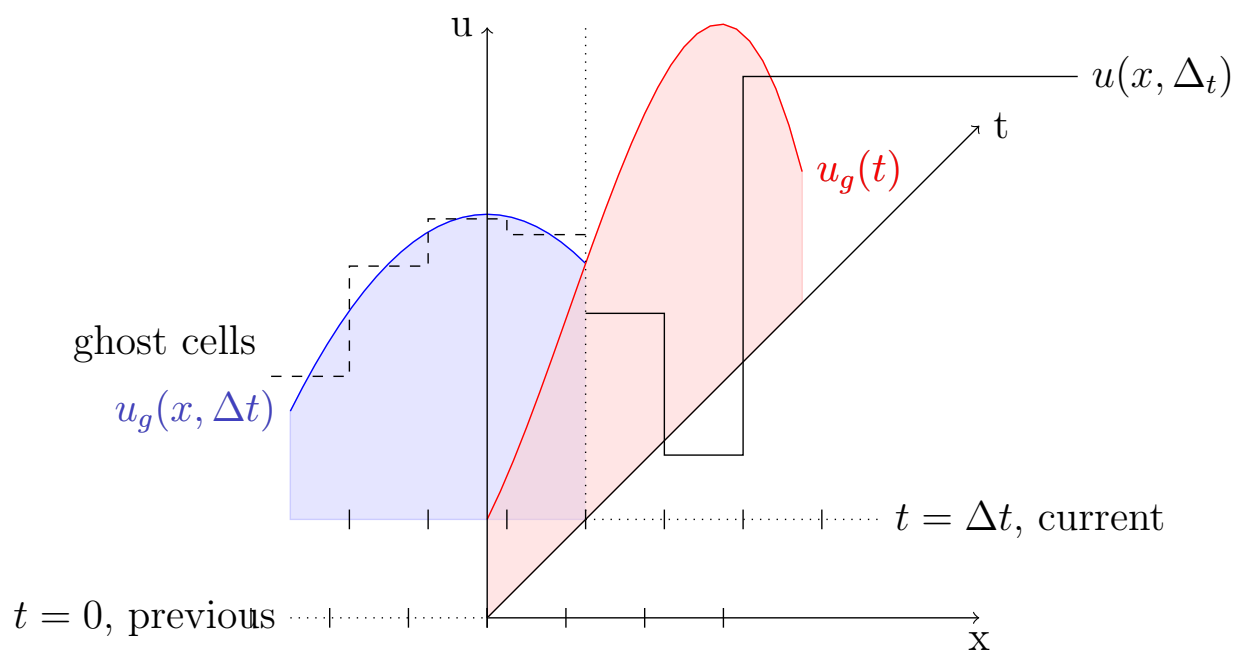

Figure 2: Illustration of the Boundary reconstruction.

\subsection{Numerical procedure}

In the following we summarize how to apply the above techniques to obtain a high order accurate numerical scheme at a junction. The following list provides an overview of the procedure.

1. Fill the ghost cells at the junction according to section 2.4.2.

2. Solve the zeroth order Riemann problem at the junction, as described in section 2.2, to obtain the Godunov states at the junction.

3. Solve the generalized Riemann problem at the junction, as described in section 2.3, to obtain the temporal derivatives of the Godunov states at the junction.

4. Compute the fluxes across cell interfaces at the junction from the Godunov states and their derivatives by formula (14).

5. Fill the ghost cells at the junction according to section 2.4.1.

6. Run a high order finite volume scheme e.g. ADER to compute the fluxes across interior cell interfaces.

In this procedure the ghost cells are filled twice, the first time for the purpose of reconstruction at the boundary to obtain Riemann data at the junction, the second time for the scheme along the edges. 


\subsection{Conservation at the junction}

Many coupling conditions state the conservation of certain quantities, e.g. in most cases a total mass is conserved as in (6) or (7). Therefore it is desirable, that the coupling procedure maintains this property for the numerical scheme. Note that a quantity is conserved across the junction, if and only if the sum over the fluxes of this quantity vanishes at the node.

Theorem 1. If the conservation of a quantity $u_{q}$ is part of the coupling conditions, i.e.

$$
\Phi_{j}\left(u^{1}, \ldots, u^{m}\right)=\sum_{i=1}^{m} f_{q}\left(u^{i}\right)=0
$$

for some $j$, then the coupling technique of section 2.5 does conserve that quantity.

Proof. Consider a single time step of the numerical procedure, i.e. the time interval $[0, \Delta t]$. For the sum of the fluxes across the junction the following computation holds

$$
\begin{aligned}
\sum_{i=1}^{n} \int_{0}^{\Delta t} f_{q}^{i}\left(u_{g}^{i}(\tau)\right) d \tau & =\left.\sum_{i=1}^{n} \int_{0}^{\Delta t} \sum_{k=0}^{k_{\max }} \frac{\tau^{k}}{k !}\left[\partial_{t}^{k} f_{q}^{i}\left(u_{g}^{i}(\tau)\right)\right]\right|_{\tau=0} d \tau \\
& =\left.\sum_{k=0}^{k_{\max }} \int_{0}^{\Delta t} \frac{\tau^{k}}{k !} d \tau \sum_{i=1}^{n} \partial_{t}^{k} f_{q}^{i}\left(u_{g}^{i}(\tau)\right)\right|_{\tau=0} \\
& =\sum_{k=0}^{k_{\max }} \int_{0}^{\Delta t} \frac{\tau^{k}}{k !} d \tau \underbrace{\left.\left[\partial_{t}^{k} \Phi_{j}\left(u_{g}^{1}(\tau), \ldots, u_{g}^{n}(\tau)\right)\right]\right|_{\tau=0}}_{=0 \text { by construction }} \\
& =0 .
\end{aligned}
$$

For a numerical method to be conservative across the node, the flux across the boundary (14) has to correspond exactly to the one in the coupling procedure. In the update formula of the ADER approach (14) no quadrature rule must be used, but the derivatives $\partial_{t}^{k} f_{q}^{i}\left(u_{g}^{i}(\tau)\right)$ should be computed in the same way as in (11). Otherwise the conservation is only given up to an error of the quadrature rule. Some small numerical errors might still arise, depending on the accuracy of the solver for the nonlinear system (8). 


\subsection{Consistency of the 2-junction}

For suitable coupling conditions, such as (6) or (7), the exact solution of a 2-junction with two identical edges coincides with the solution of one edge of combined length. In this special case we want to restore the solution of the classical ADER approach [18].

For this particular situation, we consider the two edges oriented in the same direction, such that the coupling conditions can be transformed into

$$
\Phi\left(u^{1}, u^{2}\right)=u^{1}-u^{2}=0 .
$$

In this case the generalized Riemann problem at such a 2-junction coincides with the classical generalized Riemann problem.

Theorem 2. The solution of the generalized Riemann problem at a 2-junction with the coupling conditions (16) obtained by the procedure of section $2.5 \mathrm{co}$ incides with the solution of a classical generalized Riemann problem.

To prove this theorem we need some definitions and an auxiliary lemma.

Definition 3. For an expression $P=F\left(\tilde{\partial}^{\alpha^{1}} u, \tilde{\partial}^{\alpha^{2}} u, \ldots, \tilde{\partial}^{\alpha^{k}} u\right)$ depending on partial derivatives of $u$, with

$$
u=u\left(x_{1}, x_{2}, \ldots, x_{n}\right), \quad \tilde{\partial}=\left(\partial_{x_{1}}, \partial_{x_{2}}, \ldots, \partial_{x_{n}}\right)^{T},
$$

and the multi index $\alpha^{i}=\left(\alpha_{1}^{i}, \alpha_{2}^{i}, \ldots, \alpha_{n}^{i}\right)^{T}$, the derivative order of $P$ is defined as

$$
\mathrm{O}_{\mathrm{d}}(P)=\max _{1 \leq i \leq k}\left|\alpha^{i}\right|
$$

i.e. the order of the highest order derivative in $P$.

The following simple estimates hold

$$
\begin{aligned}
& \mathrm{O}_{\mathrm{d}}\left(P_{1} \pm P_{2}\right) \leq \max \left(\mathrm{O}_{\mathrm{d}}\left(P_{1}\right), \mathrm{O}_{\mathrm{d}}\left(P_{2}\right)\right), \mathrm{O}_{\mathrm{d}}\left(P_{1} P_{2}\right) \leq \max \left(\mathrm{O}_{\mathrm{d}}\left(P_{1}\right), \mathrm{O}_{\mathrm{d}}\left(P_{2}\right)\right) \\
& \mathrm{O}_{\mathrm{d}}\left(\frac{P_{1}}{P_{2}}\right) \leq \max \left(\mathrm{O}_{\mathrm{d}}\left(P_{1}\right), \mathrm{O}_{\mathrm{d}}\left(P_{2}\right)\right), \quad \mathrm{O}_{\mathrm{d}}\left(\partial_{x_{i}} P\right) \leq \mathrm{O}_{\mathrm{d}}(P)+1
\end{aligned}
$$

Lemma 1. The result of the Cauchy-Kowalewski procedure can be written as

$$
\partial_{t}^{k} u=C K\left(u, \partial_{x} u, \ldots, \partial_{x}^{k} u\right)=(-D F(u))^{k} \partial_{x}^{k} u+R\left(u, \partial_{x} u, \ldots, \partial_{x}^{k-1} u\right),
$$

with $\mathrm{O}_{\mathrm{d}}(R) \leq k-1$. 
Proof. The proof is by induction. The cases $k=0$ and $k=1$ with $u=u$ and $u_{t}=-\nabla f(u) u_{x}$ are obvious. Assume that (18) hold for a given $k$. Differentiating (18) w.r.t. time

$$
\begin{aligned}
\partial_{t}^{k+1} u & =(-\nabla f(u))^{k} \partial_{x}^{k} \partial_{t} u+\partial_{t}\left[(-\nabla f(u))^{k}\right] \partial_{x}^{k} u+\partial_{t} R \\
\Rightarrow \partial_{t}^{k+1} u & =(-\nabla f(u))^{k+1} \partial_{x}^{k+1} u+\underbrace{(-\nabla f(u))^{k} R_{1}}_{\mathrm{O}_{\mathrm{d}} \leq k}+\underbrace{\partial_{t} R}_{\mathrm{O}_{\mathrm{d}} \leq k},
\end{aligned}
$$

and applying the estimates (17) concludes the proof of the lemma.

Proof (Proof of Theorem 2). The proof is by induction over $k$.

Denote the solution of the classical generalized Riemann problem by $u_{g}$ and that of the generalized Riemann problem at the 2 -junction by $\tilde{u}_{g}=u_{g}^{1}=u_{g}^{2}$. In the case $k=1, u_{g}=\tilde{u}_{g}$ holds due to the coupling conditions (16). For the step $k-1 \rightarrow k$ we first look at the generalized Riemann problem at the 2junction. The Riemann data is computed by the modified CK procedure (12) from the first $k$ derivatives of the Godunov state known from the previous $k-1$ steps and $\partial_{x}^{k} u_{l / r}$ respectively

$$
\begin{aligned}
& \partial_{t}^{k} U_{l}=\tilde{\mathrm{CK}}\left(\tilde{u}_{g}, \partial_{t} \tilde{u}_{g}, \ldots, \partial_{t}^{k-1} \tilde{u}_{g}, \partial_{x}^{k} u_{l}\right) \\
& \stackrel{\text { Lemma1 }}{=}\left(-\nabla f\left(u_{g}\right)\right)^{k} \partial_{x}^{k} u_{l}+R\left(\tilde{u}_{g}, \partial_{x} \tilde{u}_{g}, \ldots, \partial_{x}^{k-1} \tilde{u}_{g}\right) \\
& \partial_{t}^{k} U_{r}=\tilde{C K}\left(\tilde{u}_{g}, \partial_{t} \tilde{u}_{g}, \ldots, \partial_{t}^{k-1} \tilde{u}_{g}, \partial_{x}^{k} u_{r}\right) \\
& \stackrel{\text { Lemma1 }}{=}\left(-\nabla f\left(u_{g}\right)\right)^{k} \partial_{x}^{k} u_{r}+\underbrace{R\left(\tilde{u}_{g}, \partial_{x} \tilde{u}_{g} \ldots, \partial_{x}^{k-1} \tilde{u}_{g}\right)}_{=B} \text {. }
\end{aligned}
$$

These are the Riemann data for the linear governing equation

$$
\left(\partial_{t}^{k} u\right)_{t}+\nabla f\left(u_{g}\right)\left(\partial_{t}^{k} u\right)_{x}=0
$$

For its solution let $\lambda_{1}, \ldots, \lambda_{d}$ denote the eigenvalues corresponding to the eigenvectors $r_{1}, \ldots, r_{d}$ of $\nabla f\left(u_{g}\right)$. If we represent $\partial_{x}^{k} u_{l}$ and $\partial_{x}^{k} u_{r}$ as

$$
\partial_{x}^{k} u_{l}=\sum_{i=1}^{d} \alpha_{i} r_{i}, \quad \partial_{x}^{k} u_{r}=\sum_{i=1}^{d} \beta_{i} r_{i}
$$


we obtain by (19) the Riemann data

$$
\begin{aligned}
& \partial_{t}^{k} u_{l}=\left(-\nabla f\left(u_{g}\right)\right)^{k} \sum_{i=1}^{d} \alpha_{i} r_{i}+B=\sum_{i=1}^{d} \alpha_{i}\left(-\lambda_{i}\right)^{k} r_{i}+B \\
& \partial_{t}^{k} u_{r}=\left(-\nabla f\left(u_{g}\right)\right)^{k} \sum_{i=1}^{d} \beta_{i} r_{i}+B=\sum_{i=1}^{d} \beta_{i}\left(-\lambda_{i}\right)^{k} r_{i}+B
\end{aligned}
$$

The solution of the Riemann problem can be written as

$$
\partial_{t}^{k} \tilde{u}_{g}=\sum_{\lambda_{i}<0} \beta_{i}\left(-\lambda_{i}\right)^{k} r_{i}+\sum_{\lambda_{i}>0} \alpha_{i}\left(-\lambda_{i}\right)^{k} r_{i}+B
$$

On the other hand, for the classical Riemann problem, the initial data to the equation

$$
\left(\partial_{x}^{k} u\right)_{t}+\nabla f\left(u_{g}\right)\left(\partial_{x}^{k} u\right)_{x}=0
$$

$\partial_{x}^{k} u_{l}$ and $\partial_{x}^{k} u_{r}$ directly stem from the spatial reconstruction. Its solution is

$$
\partial_{x}^{k} u_{g}=\sum_{\lambda_{i}<0} \beta_{i} r_{i}+\sum_{\lambda_{i}>0} \alpha_{i} r_{i}
$$

This can be transformed by CK into the temporal derivative

$$
\begin{aligned}
\partial_{t}^{k} u_{g}=\operatorname{CK}\left(u_{g}, \partial_{x} u_{g}, \ldots, \partial_{x}^{k} u_{g}\right) & \\
& \stackrel{\text { Lemma }}{=}\left(-\nabla f\left(u_{g}\right)\right)^{k} \partial_{x}^{k} u_{g}+R\left(u_{g}, \partial_{x} u_{g}, \ldots, \partial_{x}^{k-1} u_{g}\right)
\end{aligned}
$$

Note that the data from the previous steps up to $l=k-1$ coincide due to the induction hypothesis, i.e. $\partial_{x}^{l} u_{g}=\partial_{x}^{l} \tilde{u}_{g}$, and thus

$$
\begin{aligned}
\partial_{t}^{k} u_{g} & =\left(-\nabla f\left(u_{g}\right)\right)^{k} \partial_{x}^{k} u_{g}+R\left(\tilde{u}_{g}, \partial_{x} \tilde{u}_{g}, \ldots, \partial_{x}^{k-1} \tilde{u}_{g}\right) \\
& =\left(-\nabla f\left(u_{g}\right)\right)^{k} \partial_{x}^{k} u_{g}+B
\end{aligned}
$$

holds. Due to $r_{i}$ being an eigenvector of $\nabla f\left(u_{g}\right)$ we know that

$$
\left(-\nabla f\left(u_{g}\right)\right)^{k} r_{i}=\left(-\lambda_{i}\right)^{k} r_{i}
$$


Inserting (20) and using (21) concludes the proof.

$$
\begin{aligned}
\partial_{t}^{k} u_{g} & =\left(-\nabla f\left(u_{g}\right)\right)^{k}\left(\sum_{\lambda_{i}<0} \beta_{i} r_{i}+\sum_{\lambda_{i}>0} \alpha_{i} r_{i}\right)+B \\
& =\sum_{\lambda_{i}<0} \beta_{i}\left(-\lambda_{i}\right)^{k} r_{i}+\sum_{\lambda_{i}>0} \alpha_{i}\left(-\lambda_{i}\right)^{k} r_{i}+B \\
& =\partial_{t}^{k} \tilde{u}_{g} .
\end{aligned}
$$

\section{Numerical examples}

In this section we study several numerical examples elucidating the performance of the presented method. As model we use the shallow water equations, i.e. (5) with the choice

$$
p(\rho)=\frac{9.81}{2} \rho^{2}
$$

thus $\rho$ denotes the depth of water and $q$ is the flow. The length of the edges is $L=1$ and as coupling conditions we use the equal height conditions (6).

As numerical method on the edges we use the ADER scheme as described in [18]. For the WENO reconstruction therein we use the classical approach [14], but for the nonlinear smoothness indicators

$$
\alpha_{r}=\frac{d_{r}}{\left(\beta_{r}+\epsilon\right)^{2}}
$$

we choose $\epsilon=10^{-14}$ in order to correctly capture small shocks. All computations are done with a spatial resolution of $n_{x}=100$ cells per edge and an adaptive time step according to a CFL number of 0.95. The nonlinear system at the junction (8) is solved with a Newton type method.

\subsection{Rates of convergence}

In the first numerical example we want to check if the expected high order of convergence can be observed numerically. Therefore we consider the simplest possible network, three edges and two nodes connected as depicted in Figure 4. As coupling conditions we use the impulse-flux coupling (7). For 
the initial data we need smooth functions along the edges, which simultaneously simultaneously fulfill all the coupling conditions at the junctions and all its temporal derivatives (11)

$$
\begin{array}{ll}
\rho_{i}(x, 0)=\frac{1}{2} \cos (2 \pi x)+1 & i=1,2,3 \\
q_{1}(x, 0)=-\left(\frac{1}{4} \cos (2 \pi x)-1 / 4\right), & \\
q_{i}(x, 0)=-\left(\frac{1}{8} \cos (2 \pi x)-1 / 8\right) & i=2,3 .
\end{array}
$$

We compute the solution up to $t=0.05$, such that all functions remain sufficiently smooth. The solution and the evaluation of the convergence in the $L^{1}$-norm is shown in Figure 3 . We can see that up to order five the expected order of convergence is fully reached, whereas for even higher orders only smaller improvements can be observed. This can be explained with the the poor condition number of the inverse Cauchy Kowalevski procedure for big input data, e.g. data obtained from a CK transformation. At least in our implementation the condition number increases notably when considering orders higher than five.
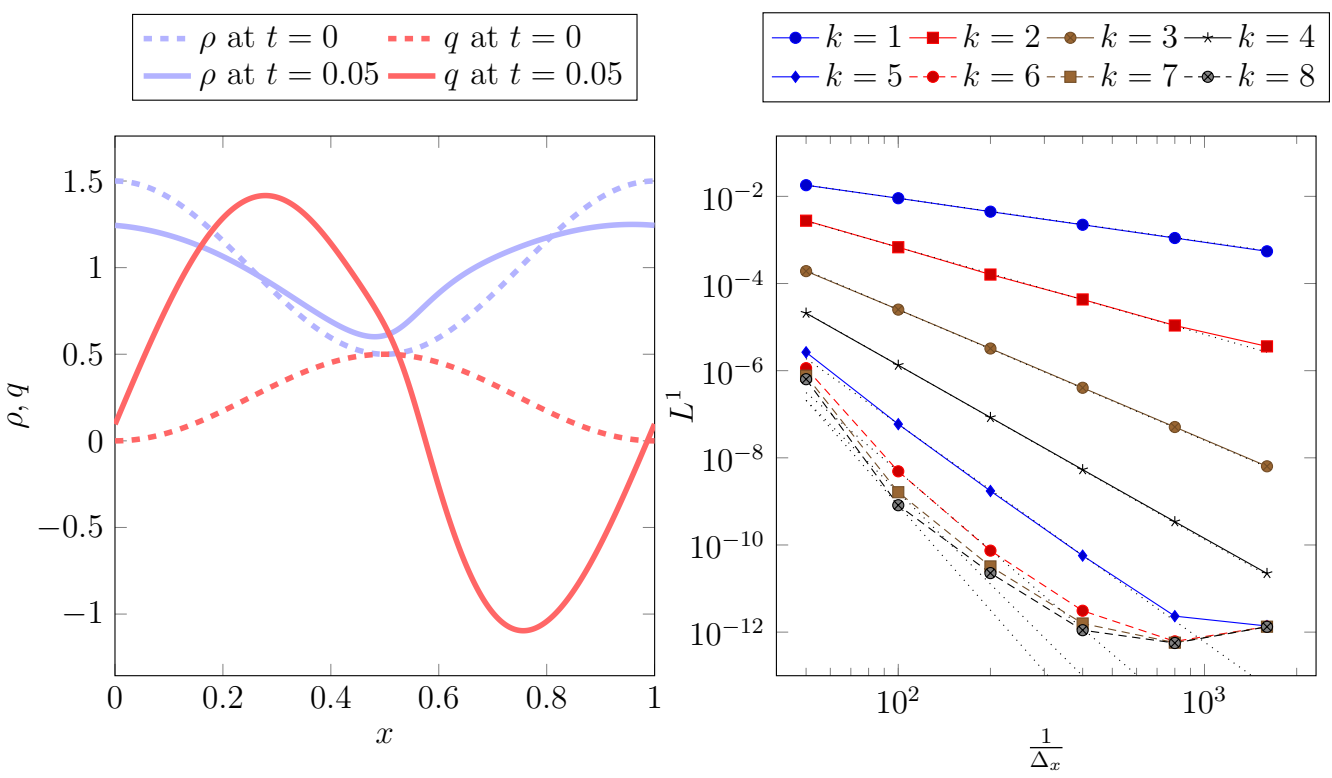

Figure 3: Initial values and error in the $L^{1}$ norm at $t=0.05$ on $E_{1}$. 


\subsection{High order schemes and low order coupling}

In this example we want to highlight the importance of treating the coupling conditions accurately. Therefore we again consider the simple network depicted in Figure 4 with constant initial data on the two parallel edges and a smooth profile on the first edge $E_{1}$

$$
\rho_{1}(x, 0)=\tilde{p}(x), \quad \rho_{2,3}(x, 0)=\frac{1}{2}, \quad q_{i}(x, 0)=0 \quad \forall i=1, \ldots, 3,
$$

with $\tilde{p}$ being the polynomial of degree 16 defined by

$$
\tilde{p}(0.5)=1, \quad \partial_{x}^{k} \tilde{p}(0)=\partial_{x}^{k} \tilde{p}(1)=0 \quad \forall k=1, \ldots, 8 .
$$

For this data we compare a scheme of order $k=6$ with high order coupling, $k_{c}=6$, to the same scheme where the ghost cells are only filled by constant data, corresponding to a first order coupling procedure, i.e. $k_{c}=1$. As reference solutions we consider a completely first order scheme and the 6th order scheme with high order coupling with a spatial resolution of $n_{x}=500$ grid cells per edge. In Figure 5 the values of $\rho$ at $t=5$ on edge $E_{1}$ are plotted.

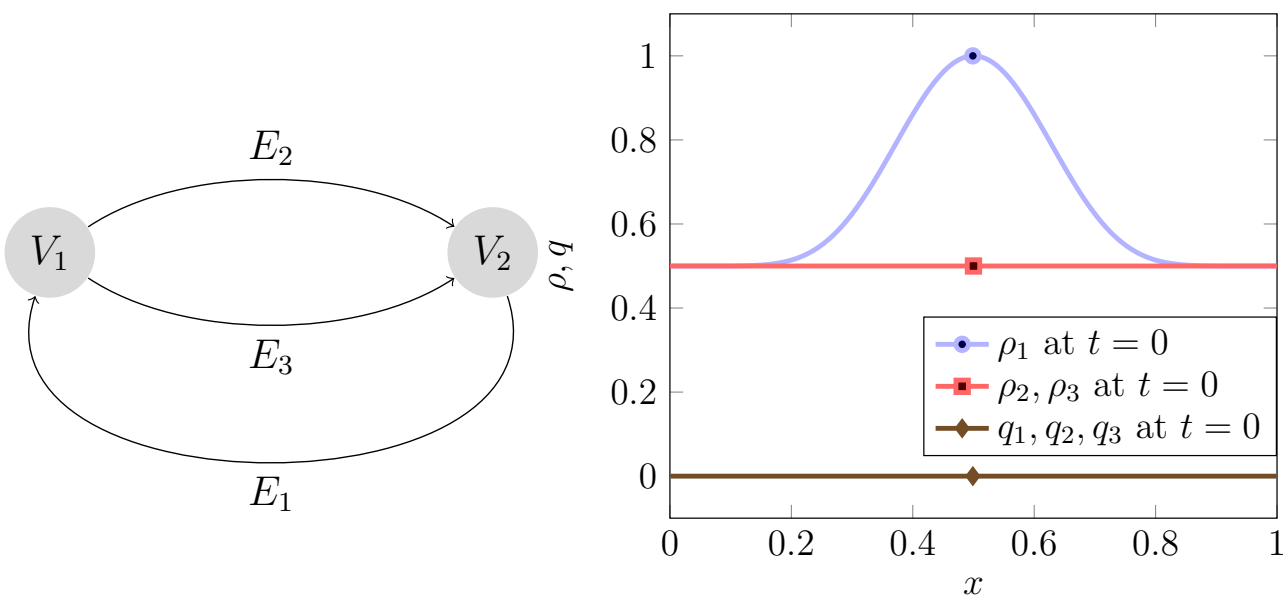

Figure 4: Split circle network and initial values.

All schemes accurately capture two shocks, but the solution also incorporates many additional waves emerging from many wave-junction interactions during $t=0$ and $t=5$. 
We can clearly see that the solution of the high order scheme with the low order coupling deviates from the two reference solutions, whereas the the scheme with the high order coupling procedure has a very good agreement with the solutions on the fine grid. Furthermore we can observe that the high resolution scheme on the coarse grid is able to capture more waves than the first order scheme on the fine grid.

\subsection{Shock-propagation on a big network}

In these last test cases we want to show that the high order coupling procedure is stable if shock waves are passing the junction and fast enough to be applied on large networks. Therefore we consider a closed network of 128 edges and 96 nodes as depicted in Figure 6. As initial data we adapt the benchmark of [32], such that

$$
\begin{aligned}
& \rho_{1}(x, 0)=\left\{\begin{array}{ll}
1+o & x<\frac{3}{4} \\
1 & x \geq \frac{3}{4}
\end{array} \quad \rho_{2}(x, 0)=1+o\right. \\
& \rho_{i}(x, 0)=1 \forall i=3, \ldots, 128
\end{aligned}
$$

with the free parameter $o$. In Figure 7 we compare the solutions of schemes of orders $k=1, k=4$ and $k=6$ for a large initial jump $o=1$ at time $t=3.1$ on edge $E_{127}$. We can see two shock waves, the first arising from the initial Riemann problem, whereas the upper shock wave results from a later interaction with a junction. We can clearly see that the higher order methods accurately capture both shocks, while for the first order method the second shock suffers from strong numerical diffusion. It is important to note that this first shock wave arises from the initial shock wave of the Riemann problem on $E_{1}$, which is split into many much smaller shocks and builds up by several shock-shock interactions at the merging junctions. We can not report any notable numerical oscillations arising from the coupling procedure.

Similar results we obtain for a test with a much smaller shock of $o=$ 0.001. Figure 8 shows the solutions for $\rho$ on $E_{127}$ at $t=3.4$. The shock is resolved sharply by the higher order schemes, whereas the first order solution is smeared over one third of the edge. Again, the shock builds up from many even smaller shocks by several simultaneous shock-shock interactions at the merging junctions. This shows that the higher order coupling procedure is capable to accurately resolve very fine structures within the solution. 


\section{Concluding remarks}

We have presented a numerical procedure for high order accurate treatment of coupled hyperbolic conservation laws. Several numerical examples show that this coupling technique can be used to build accurate, fast and stable numerical methods for networks of conservation laws. Future developments are aiming to extend this approach to the coupling of conservation laws with ordinary differential equations at the boundary.

[1] M. Á. Fernández, V. Milišić, A. Quarteroni, Analysis of a geometrical multiscale blood flow model based on the coupling of ODEs and hyperbolic PDEs, Multiscale Model. Simul. 4 (1) (2005) 215-236 (electronic).

[2] V. Casulli, M. Dumbser, E. F. Toro, Semi-implicit numerical modeling of axially symmetric flows in compliant arterial systems, Int. J. Numer. Methods Biomed. Eng. 28 (2) (2012) 257-272.

[3] J. Alastruey, K. H. Parker, J. Peiró, S. J. Sherwin, Lumped parameter outflow models for 1-D blood flow simulations: effect on pulse waves and parameter estimation, Commun. Comput. Phys. 4 (2) (2008) 317-336.

[4] E. T. L.O. Müller, A global multi-scale mathematical model for the human circulation with emphasis on the venous system.

[5] M. Herty, V. Sachers, Adjoint calculus for optimization of gas networks, Netw. Heterog. Media 2 (4) (2007) 733-750.

[6] M. K. Banda, M. Herty, A. Klar, Coupling conditions for gas networks governed by the isothermal Euler equations, Netw. Heterog. Media 1 (2) (2006) 295-314.

[7] J. Brouwer, I. Gasser, M. Herty, Gas pipeline models revisited: model hierarchies, nonisothermal models, and simulations of networks, Multiscale Model. Simul. 9 (2) (2011) 601-623.

[8] E. J. P. G. Schmidt, On junctions in a network of canals, in: Control theory of partial differential equations, Vol. 242 of Lect. Notes Pure Appl. Math., Chapman \& Hall/CRC, Boca Raton, FL, 2005, pp. 207212. 
[9] R. Borsche, R. M. Colombo, M. Garavello, On the coupling of systems of hyperbolic conservation laws with ordinary differential equations, Nonlinearity 23 (11) (2010) 2749-2770.

[10] R. Borsche, Modeling and simulation of sewer networks and coupled surface flow, Ph.D. thesis, University of Kaiserslautern (2011).

[11] A. S. Leon, Improved modeling of unsteady free surface, pressurized and mixed flows in storm-sewer systems, Ph.D. thesis, University of Illinois at Urbana-Champaign (2007).

[12] G. M. Coclite, M. Garavello, B. Piccoli, Traffic flow on a road network, SIAM journal on mathematical analysis 36 (6) (2005) 1862-1886.

[13] M. Garavello, B. Piccoli, Traffic flow on networks, Vol. 1 of AIMS Series on Applied Mathematics, American Institute of Mathematical Sciences (AIMS), Springfield, MO, 2006, conservation laws models.

[14] G.-S. Jiang, C.-W. Shu, Efficient implementation of weighted eno schemes., Tech. rep., DTIC Document (1995).

[15] C. Hu, C.-W. Shu, Weighted essentially non-oscillatory schemes on triangular meshes, Journal of Computational Physics 150 (1) (1999) 97-127.

[16] J. Qiu, C.-W. Shu, On the construction, comparison, and local characteristic decomposition for high-order central weno schemes, Journal of Computational Physics 183 (1) (2002) 187-209.

[17] M. Dumbser, M. Käser, Arbitrary high order non-oscillatory finite volume schemes on unstructured meshes for linear hyperbolic systems, Journal of Computational Physics 221 (2) (2007) 693-723.

[18] E. F. Toro, Riemann solvers and numerical methods for fluid dynamics: a practical introduction, Springer, 2009.

[19] T. Aboiyar, E. H. Georgoulis, A. Iske, Adaptive ADER methods using kernel-based polyharmonic spline WENO reconstruction, SIAM J. Sci. Comput. 32 (6) (2010) 3251-3277.

[20] M. Dumbser, C. Enaux, E. F. Toro, Finite volume schemes of very high order of accuracy for stiff hyperbolic balance laws, J. Comput. Phys. 227 (8) (2008) 3971-4001. 
[21] C.-W. Shu, High order weighted essentially nonoscillatory schemes for convection dominated problems, SIAM review 51 (1) (2009) 82-126.

[22] M. Dumbser, M. Castro, C. Parés, E. F. Toro, Ader schemes on unstructured meshes for nonconservative hyperbolic systems: Applications to geophysical flows, Computers \& Fluids 38 (9) (2009) 1731-1748.

[23] G. Bretti, R. Natalini, B. Piccoli, Numerical algorithms for simulations of a traffic model on road networks, J. Comput. Appl. Math. 210 (1-2) (2007) 71-77.

[24] M. Gugat, Optimal nodal control of networked hyperbolic systems: evaluation of derivatives, Adv. Model. Optim. 7 (1) (2005) 9-37 (electronic).

[25] O. Kolb, Simulation and optimization of gas and water supply networks, Ph.D. thesis, University of Darmstadt (2011).

[26] R. M. Colombo, G. Guerra, On general balance laws with boundary, J. Differential Equations 248 (5) (2010) 1017-1043.

[27] R. M. Colombo, M. Garavello, On the Cauchy problem for the $p$-system at a junction, SIAM J. Math. Anal. 39 (5) (2008) 1456-1471.

[28] R. M. Colombo, M. Garavello, A well posed Riemann problem for the $p$-system at a junction, Netw. Heterog. Media 1 (3) (2006) 495-511.

[29] R. M. Colombo, M. Herty, V. Sachers, On $2 \backslash$ times2 conservation laws at a junction, SIAM Journal on Mathematical Analysis 40 (2) (2008) 605-622.

[30] S. Tan, C.-W. Shu, Inverse lax-wendroff procedure for numerical boundary conditions of conservation laws, Journal of Computational Physics 229 (21) (2010) 8144-8166.

[31] C. E. Castro, E. F. Toro, Solvers for the high-order Riemann problem for hyperbolic balance laws, J. Comput. Phys. 227 (4) (2008) 2481-2513.

[32] R. J. LeVeque, Balancing source terms and flux gradients in highresolution Godunov methods: the quasi-steady wave-propagation algorithm, J. Comput. Phys. 146 (1) (1998) 346-365. 

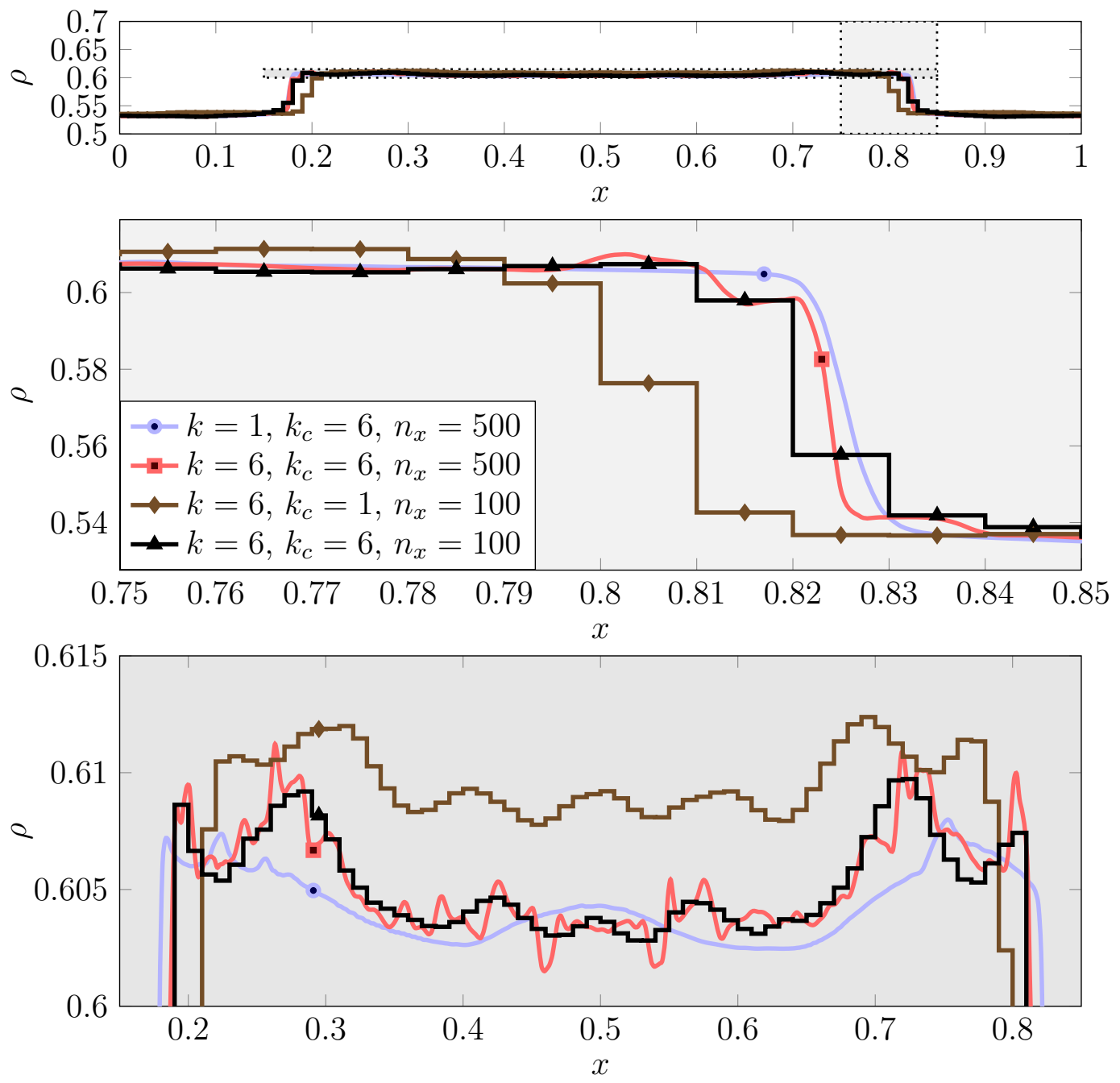

Figure 5: Numerical solutions for different orders at $t=5$. 


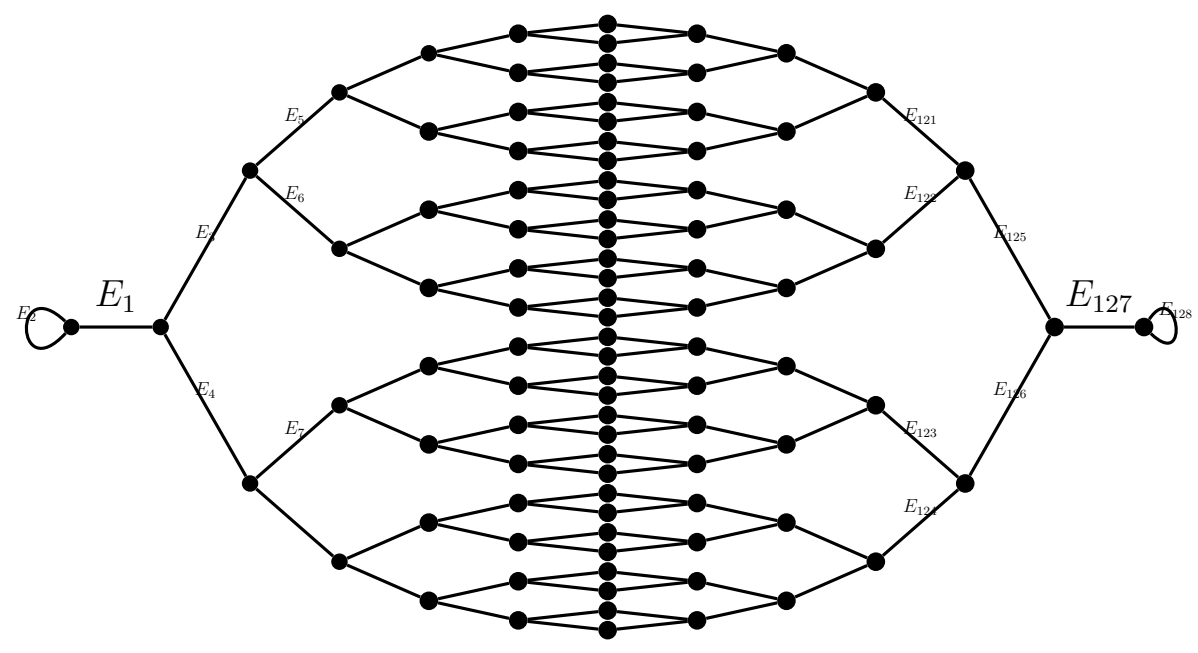

Figure 6: Split and join network of depth 6 .
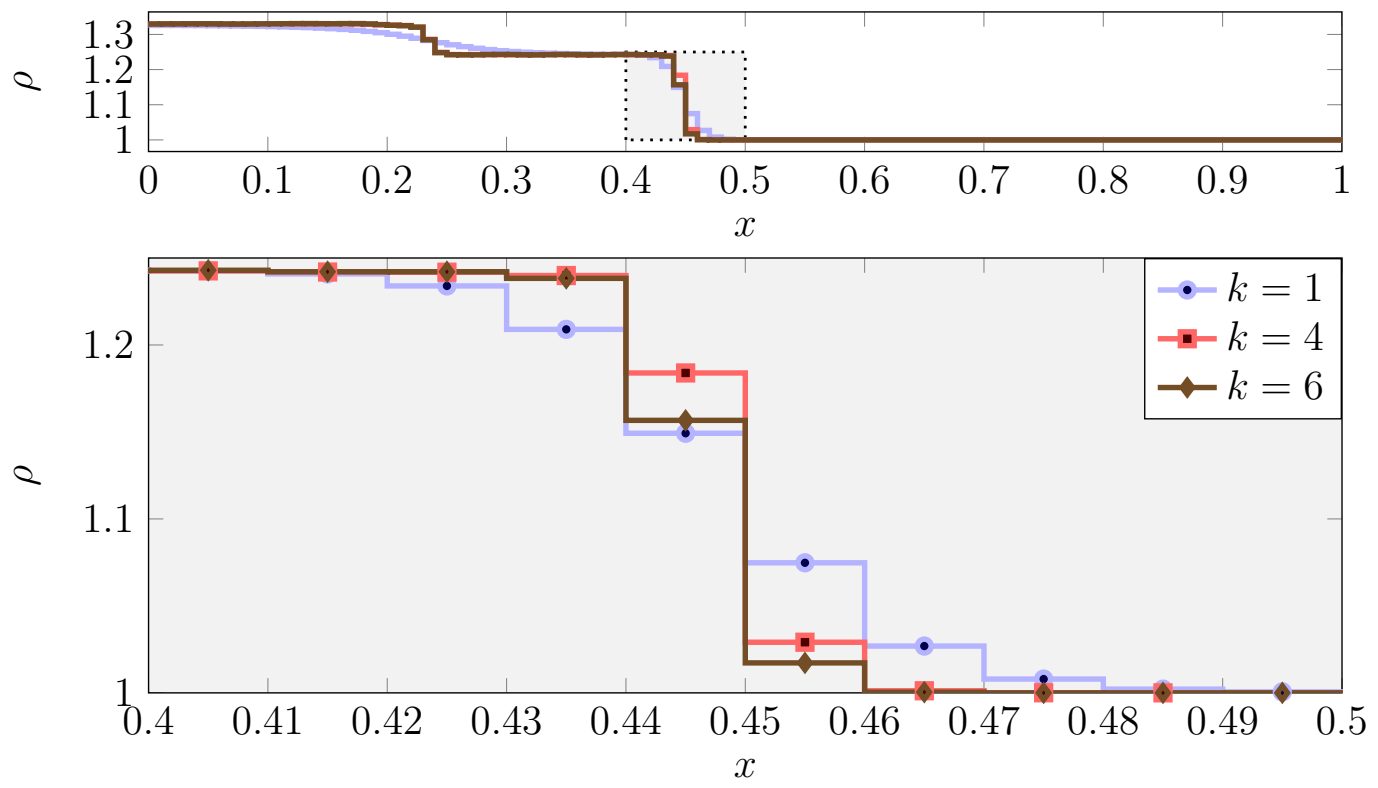

Figure 7: Large shock: $\rho$ on $E_{127}$ at $t=3.1$. 

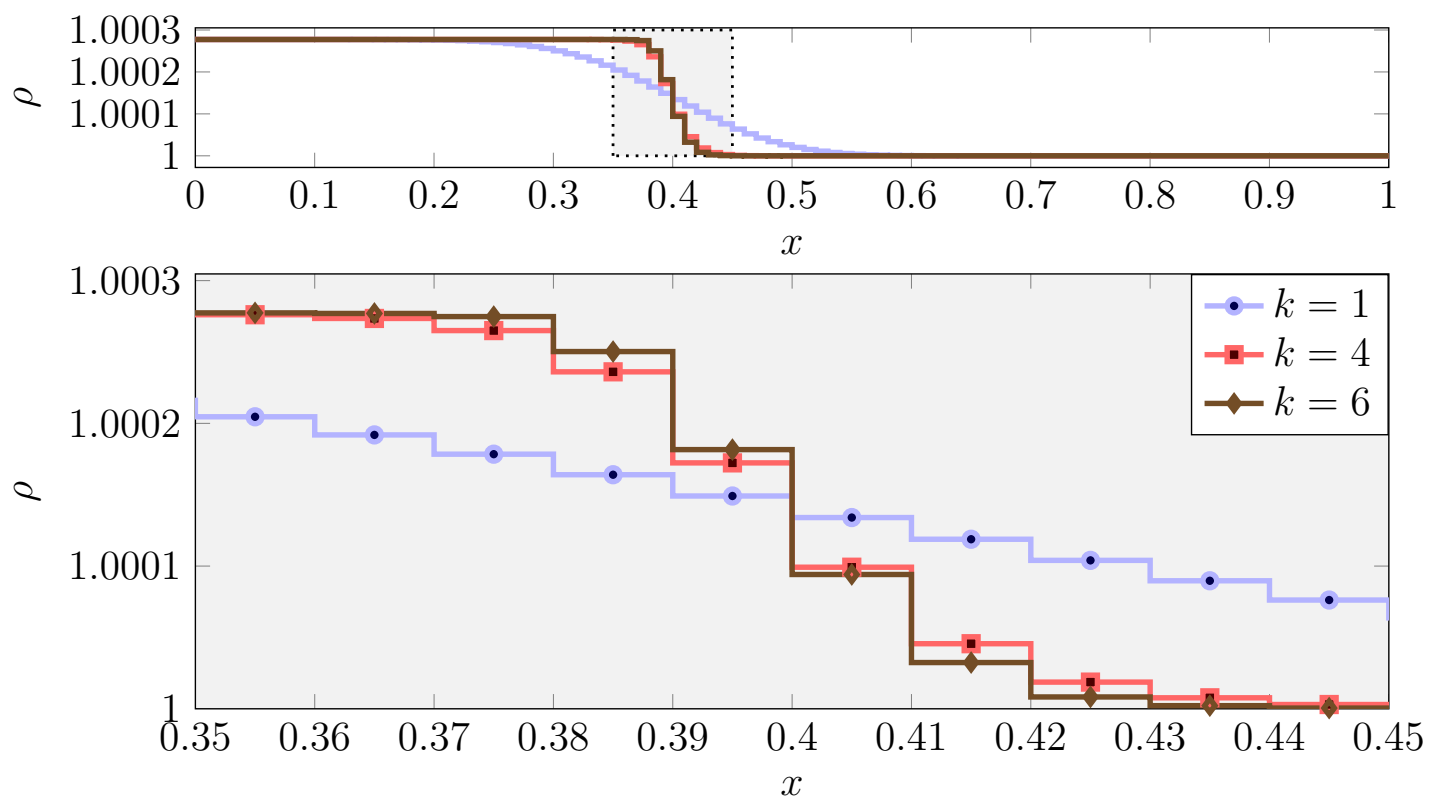

Figure 8: Small shock: $\rho$ on $E_{127}$ at $t=3.4$. 